\title{
Statistics of Raman-active excitations via measurement of Stokes-anti-Stokes correlations
}

\author{
Özgür E. Müstecaplıoğlu and Alexander S. Shumovsky \\ Department of Physics, Bilkent University, Bilkent, 06533 Ankara, Turkey
}

(Received 9 November 1998; revised manuscript received 1 March 1999)

\begin{abstract}
A general fundamental relation connecting the correlation of Stokes and anti-Stokes modes to the quantum statistical behavior of vibration and pump modes in Raman-active materials is derived. We show that under certain conditions this relation can be used to determine the equilibrium number variance of phonons. Time and temperature ranges for which such conditions can be satisfied are studied and found to be available in today's experimental standards. Furthermore, we examine the results in the presence of multimode pump as well as for the coupling of pump to the many vibration modes and discuss their validity in these cases.
\end{abstract}

[S0163-1829(99)00730-4]

\section{INTRODUCTION}

The concept of squeezed state has been established in the language of physics mainly by the developments in quantum optics. On the other hand, a basic requirement of finding a system in a squeezed state is to have bosons as the constituents of the system interacting in a pairwise manner and that might be fulfilled not only in optical systems but in some other Bose-type systems as well. In actual fact, the introduction of squeezed states in optics ${ }^{1}$ was based on the previous consideration of superfluidity ${ }^{2}$ in liquid ${ }^{4} \mathrm{He}$ (also see Ref. 3 ). While the squeezing of quantum fluctuations is the most well-known aspect of squeezed states, a rich variety of effects might be expected due to their interesting statistical properties even at thermal equilibrium. Certain effects like antibunching have already been observed in the realm of quantum optics and this makes it an intriguing question how to find squeezed states and their effects in other places. In this context, few proposals have been suggested for the generation and detection of squeezed states of Bose-type excitations in solids. ${ }^{4-6}$ Quite recently, squeezed phonons have been produced and detected. ${ }^{7}$

It is very interesting that, unlike the case of light, the squeezed states of phonons may arise from different microscopic interactions in solids even at thermal equilibrium. ${ }^{8}$ Deviations from typical equilibrium distribution of phonons, namely, Bose-Einstein distribution, might arise from anharmonic interactions among phonons or from some other mechanisms, such as the polariton coupling in ionic crystals $^{4,9}$ or polaron mechanism. ${ }^{10}$ In such cases, the equilibrium distribution of phonons is that of squeezed thermal phonons. ${ }^{11}$ Therefore, it seems to be an important question how to determine the equilibrium distribution of phonons when there is a possibility that phonons can be found to be in nonclassical states. As a particular example of some considerable interest, the squeezed states of phonons due to the photon-optical phonon interaction in an ionic crystal ${ }^{9}$ should be mentioned here. The polariton coupling in such a system is described by the following Hamiltonian: ${ }^{12}$

$$
H=\frac{1}{2} \sum_{k} H_{k}
$$

where $\omega_{k}$ is the photon frequency, $\omega_{b}$ is the frequency of transversal oscillations of optical phonons, $g_{k}$ is the polariton coupling constant, and the operators $a_{k}, b_{k}$ describe the annihilation of photons and optical phonons, respectively. Since the Hamiltonian under consideration is the Hermitian bilinear form, it can be diagonalized by the Bogolubov canonical transformation ${ }^{2}$ similar to that used in the definition of squeezed states. ${ }^{1}$ As a result, the thermal equilibrium state of the system is described by the following density matrix:

$$
\rho(\beta)=\frac{e^{-\beta H_{p}}}{\operatorname{Tr} e^{-\beta H_{p}}},
$$

where $H_{p}$ denotes the Hamiltonian $H$ in diagonal (polariton) representation and $\beta$ is the reciprocal temperature. In analogy to the quantum optics, consider the so-called degree of coherence, ${ }^{13}$

$$
G^{(2)}=\frac{\left\langle b^{\dagger 2} b^{2}\right\rangle}{\left\langle b^{\dagger} b\right\rangle},
$$

where \langle\rangle denotes the average with respect to the density matrix $\rho(\beta)$. It is straightforward to calculate $G^{(2)}$ as a function of temperature for typical parameters of an ionic crystal (see Fig. 1). One can see that, at low temperatures, $G^{(2)}$ $\approx 8$, while the same correlation function calculated with the Bose-Einstein distribution gives $G_{B E}^{(2)}=2$. It is also seen that the strong quantum fluctuations can be observed only below $T \sim 50 \mathrm{~K}$ because they are eroded by thermal fluctuations with the increase of temperature.

In contrast to the case of nonclassical states of photons there is no efficient direct method of measurement allowing the characterization of the quantum state of Bose-type excitations in solids. ${ }^{5}$ Even though correlation functions to any order would be demanded to describe fully a quantum state, it is usually good enough to distinguish quantum states by their number variances. ${ }^{13}$ Here, we present a way to determine the number variance of phonons at equilibrium in a Raman-active medium. It is already suggested that correla- 


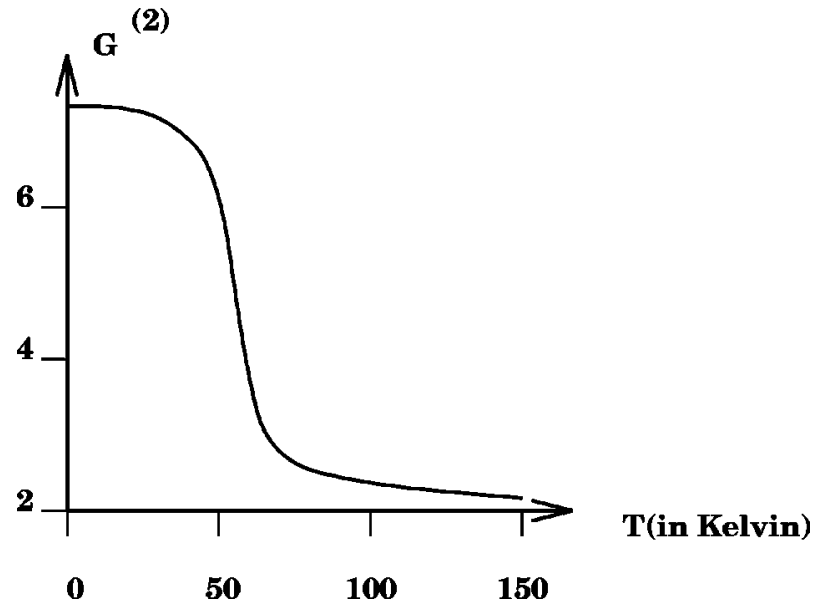

FIG. 1. Phonon degree of coherence $G^{(2)}$ versus temperature for typical parameters of an ionic crystal: $\Omega=200 \mathrm{~K}, g=25 \mathrm{~K}$.

tion Raman spectroscopy may be used to measure the quantum statistical properties of a vibration mode for the case of Stokes $(S)$-type Raman scattering through a measurement of the intensity and the Mandel's $Q$ factor of the Rayleigh mode. ${ }^{15}$ However, even at low temperatures vacuum fluctuations of the anti-Stokes (AS) modes might disturb measurements of high-order correlations, and thus careful study of the role of the AS modes in such measurements is demanded. In this paper, we follow a similar ideology in more general terms by examining both the $S$ and AS components of multimode Raman scattering. Even though the problem becomes analytically intractable when AS modes are included, it is now possible to establish an interesting connection between the number variance of phonons and the correlations of $S$ and AS modes. Moreover, due to the removing low-temperature restriction in the exclusion of AS modes, influence of temperature in the high-order quantum correlations can be examined as well.

The paper is outlined as follows. In Sec. II, using a general model of Raman-type three-body scattering, we find the intermode correlation function of $S$ and AS modes. Discussion of this general result under standard approximations of Raman scattering, with an emphasis of modifications in their range of validity, is the subject of Sec. III. Finally, Sec. IV gives a brief summary of our results and conclusions.

\section{CORRELATION OF STOKES AND ANTI-STOKES PHOTONS}

General relations between the correlation function of $S$ and AS modes and the number variance of phonons are developed in this section for the following Raman-type Hamiltonian,

$$
\begin{aligned}
H= & \sum_{\mathbf{k} \lambda} \omega_{\mathbf{k} \lambda} a_{\mathbf{k} \lambda}^{\dagger} a_{\mathbf{k} \lambda}+\sum_{\mathbf{k k}^{\prime} \mathbf{q}}\left(M_{\mathbf{k k}^{\prime} \mathbf{q}}^{S} a_{\mathbf{k}^{\prime} S}^{\dagger} a_{\mathbf{k} R} a_{\mathbf{q} V}^{\dagger}\right. \\
& \left.+M_{\mathbf{k k}^{\prime} \mathbf{q}^{\prime}}^{A} a_{\mathbf{k}^{\prime} A}^{\dagger} a_{\mathbf{k} R} a_{\mathbf{q} V}+\text { H.c. }\right),
\end{aligned}
$$

where $a_{\mathbf{k} \lambda}^{\dagger}\left(a_{\mathbf{k} \lambda}\right)$ are the creation (annihilation) operators for the $\lambda$ mode with momentum $\mathbf{k}$ and corresponding frequency $\omega_{\mathbf{k} \lambda}$. Here the mode index $\lambda=S, A, V, R$ stands for Stokes, anti-Stokes, vibration, and Rayleigh modes, respectively. As usual, the polarization labels are suppressed within the momentum symbols for the sake of notational simplicity. Coupling constants are denoted by $M_{\mathbf{k k}^{\prime} \mathbf{q}}^{S}$ for the $S$-type scattering and $M_{\mathbf{k k}^{\prime} \mathbf{q}}^{A}$ for the AS-type scattering. While writing this trilinear bosonic Hamiltonian we assumed as usual ${ }^{17}$ that the Raman scattering is observed under the condition $\omega_{R, S, A}$ $\gg \omega_{V}$ when the pairwise creation of radiation modes has quite small probability so that energy is conserved. This supposition is equivalent to the rotating-wave approximation of the quantum optics. ${ }^{16} \mathrm{We}$ also assumed that the radiation consists of three $R, S$, and AS pulses that are well separated on the frequency domain so that $\left[a_{\mathbf{k} \lambda}, a_{\mathbf{k}^{\prime} \lambda^{\prime}}^{\dagger}\right]=\delta_{\mathbf{k k}^{\prime}} \delta_{\lambda \lambda^{\prime}}$. If a single-mode strong coherent (classical) pumping is assumed, all one can expect is that the phase-matching conditions would have limited the number of active phonon modes to one. Nevertheless, it seems to be reasonable to consider the Raman scattering by an infinite Markoffian system of phonons. ${ }^{18,19}$ In particular, it permits oneself to take into account the broadening of $S$ and AS lines. The usual selection rules of Raman scattering, namely, phase-matching or quasiresonance conditions, ${ }^{17}$ are not essential for the derivation of the general relations below. Therefore, the results given in this section are also valid in not so perfect Raman coupling situations that should be important in real materials.

If we define the number operator $n_{\mathbf{k} \lambda}$ for the $\lambda$ mode with momentum $\mathbf{k}$ as $n_{\mathbf{k} \lambda}=a_{\mathbf{k} \lambda}^{\dagger} a_{\mathbf{k} \lambda}$, then the total number operator $N_{\lambda}$ for $\lambda$ mode becomes $N_{\lambda}=\Sigma_{\mathbf{k}} n_{\mathbf{k} \lambda}$. Heisenberg equations of motion yield the conservation laws, also known as Manley-Rowe relations, ${ }^{17}$

$$
\begin{aligned}
& N_{S}+N_{A}+N_{R}=C_{1}, \\
& N_{S}-N_{A}-N_{V}=C_{2} .
\end{aligned}
$$

Here constant operators $C_{1}, C_{2}$ are specified by the initial conditions. Similar relations can also be constructed for the scattering of photons of a monochromatic laser beam from a dispersionless optical phonon. ${ }^{20,8}$ Solving these equations for $N_{S}$ and $N_{A}$, the $S$ and AS correlation function is found to be

$$
\begin{aligned}
\left\langle N_{A} ; N_{S}\right\rangle= & \frac{1}{4}\left[V\left(C_{1}\right)-V\left(C_{2}\right)+V\left(N_{R}\right)-V\left(N_{V}\right)\right. \\
& \left.-2\left\langle C_{1} ; N_{R}\right\rangle-2\left\langle C_{2} ; N_{V}\right\rangle\right]
\end{aligned}
$$

where the correlation function $\langle A ; B\rangle$ of two operators $A, B$ is defined by

$$
\langle A ; B\rangle=\langle A B\rangle-\langle A\rangle\langle B\rangle,
$$

and hence variance of operator $A$ is given by the selfcorrelation function $V(A)=\langle A ; A\rangle$. Here the averages \langle\rangle are with respect to the initial state, since the Heisenberg picture is used. It is natural to consider an initial state in which the $S$ and AS modes are in their vacuum states when we obtain,

$$
\begin{aligned}
\left\langle N_{A}(t) ; N_{S}(t)\right\rangle= & \frac{1}{4}\left\{V\left[N_{R}(0)\right]-V\left[N_{V}(0)\right]+V\left[N_{R}(t)\right]\right. \\
& -V\left[N_{V}(t)\right]-2\left\langle N_{R}(0) ; N_{R}(t)\right\rangle \\
& \left.-2\left\langle N_{V}(0) ; N_{V}(t)\right\rangle\right\} .
\end{aligned}
$$


An operator $A$ at time $t$ is indicated by $A(t)$ while initially by $A(0)$. That equation connects the $S$ and AS correlation function to the quantum statistical behavior of phonons and pump photons.

Within conventional Raman theory quantum properties of pump are usually neglected through the classical pump assumption. $^{21,22}$ This approximation introduces a time range to the problem during which changes in the pump intensity remains negligible. We can apply a similar approximation by assuming an intense laser pump with photons in coherent states and performing a mean-field average over them in the above equations. Under this assumption, the correlation function of the $S$ and AS modes is related only to phonon statistics and the initial, known, number variance of the pump photons. However, time range of validity for the parametric approximation should be modified in our case. As we shall show in the subsequent section, statistical behavior of the pump might change significantly in shorter time than the occurrence of a significant change in its intensity. Our purpose is to examine the equilibrium statistics of phonons determined by $V\left[N_{V}(0)\right]$; therefore we need to express all time-dependent terms on the right-hand side of Eq. (4) in terms of initial operators to see any further relation between the $S$ and AS correlation function and the equilibrium variance of phonons. For that aim we specify a model system and study its dynamics.

We conclude this section by noting that a similar relation can be derived for the molecular Raman model, which is equivalent to the full bosonic Raman model under the Holstein-Primakoff approximation in the case of lowexcitation density. ${ }^{23}$ In that case, $S$ and AS correlations depend on the quantum statistics of population distributions of the molecular energy levels.

\section{DISCUSSIONS FOR PARAMETRIC RAMAN MODEL}

In reality, coupling of one vibration mode to the pump beam for a sufficiently long time of measurement is not an easy task. Therefore, in this section we investigate a Raman scattering in which coupling of pump photons to all phonon modes are allowed. We shall treat the pump as an intense coherent beam of photons and thus its state $\left|\psi_{R}\right\rangle$, in general, is described by a multimode coherent state,

$$
\left|\psi_{R}\right\rangle=\prod_{l} \otimes\left|\alpha_{l}\right\rangle
$$

in which $\alpha_{l}$ are the coherence parameters of the modes $\boldsymbol{l}$. According to the remarks at the end of the previous section, we now perform mean-field averaging with respect to pump photon states in Eq. (1), assuming the Raman-active material is placed in an ideal cavity that selects single modes for $S$ and AS radiations, namely $\mathbf{k}^{\prime}=\mathbf{k}_{\mathbf{A}, \mathbf{S}}$. Then after dropping constant terms, the Hamiltonian in Eq. (1) reduces to an effective one,

$$
\begin{aligned}
H^{e f f}= & \sum_{\lambda=S, A} \omega_{\lambda} n_{\lambda}+\sum_{\mathbf{q}} \omega_{\mathbf{q} V} a_{\mathbf{q} V}^{\dagger} a_{\mathbf{q} V} \\
& +\sum_{\mathbf{q}}\left(g_{\mathbf{q}}^{S} a_{S}^{\dagger} a_{\mathbf{q} V}^{\dagger}+g_{\mathbf{q}}^{A} a_{A}^{\dagger} a_{\mathbf{q} V}+\text { H.c. }\right),
\end{aligned}
$$

where new effective coupling constants $g_{\mathbf{q}}^{A, S}$ are introduced by

$$
g_{\mathbf{q}}^{A, S}=\sum_{\mathbf{k}} M_{\mathbf{k k}_{\mathbf{A}, \mathbf{S}} \mathbf{q}}^{A, S} \alpha_{\mathbf{k}}
$$

The summation above can be calculated once the density of states for the pump is also specified. As one can see, the Hamiltonian will be in the given form, involving summations over phonon modes, in all cases except the case of perfectly phase-matched single pump and phonon modes. In order to make sure that our results are not too susceptible to any imperfectness of the system arising from the multimode nature of pump or phase mismatches among the phonon and photon modes, we shall treat the problem using the model described by the above Hamiltonian involving summations over phonon modes. When finite number of phonon modes are assumed, which is reasonable for real crystals of finite size, then such a model becomes integrable since the dynamics is ruled by the following closed set of operator linear differential equations:

$$
\begin{gathered}
i \frac{d}{d t} a_{\mathbf{q} V}=\omega_{\mathbf{q} V} a_{\mathbf{q} V}+g_{\mathbf{q}}^{S} a_{S}^{\dagger}+g_{\mathbf{q}}^{A *} a_{A}, \\
i \frac{d}{d t} a_{S}^{\dagger}=-\omega_{S} a_{S}^{\dagger}-\sum_{\mathbf{q}} g_{\mathbf{q}}^{S *} a_{\mathbf{q} V}, \\
i \frac{d}{d t} a_{A}=\omega_{A} a_{A}+\sum_{\mathbf{q}} g_{\mathbf{q}}^{A} a_{\mathbf{q} V} .
\end{gathered}
$$

Let us introduce a vector of operators such that $Y$ $=\left[a_{S}^{\dagger}, a_{A},\left\{a_{\mathbf{q} V\}}\right\}\right]^{T}$. We denote the matrix of coefficients in the above set of equations by $M$ and its diagonalizing matrix by $D$, so that $D^{-1} M D=E \mathbf{1}$ with eigenvalues $E$. Thus, we get

$$
Y_{i}(t)=D_{i j} D_{j k}^{-1} Y_{k}(0) \exp \left(-i E_{j} t\right),
$$

where summation over repeated index is implied. It is therefore possible to write the solution for $\lambda=S, A$ modes in the form,

$$
a_{\lambda}(t)^{\dagger}=u_{\lambda}(t) a_{S}^{\dagger}+\mathrm{v}_{\lambda}(t) a_{A}+\sum_{\mathbf{q}} w_{\mathbf{q} \lambda}(t) a_{\mathbf{q} V} .
$$

Operators without time arguments are taken at $t=0$. Timedependent parameters $u, \mathrm{v}, w$ are determined by the elements of matrix $D$ and eigenvalues $E$. Let us note here that some general relations exists among $u, \mathrm{v}, w$ due to the commutation relations for $a_{\lambda}$ operators and they are not independent of each other. A more explicit way of evaluating $u, \mathrm{v}, w$ is presented below for the single mode phonon case where vector $Y$ reduces to three dimensions in operator space. When there are no scattered light modes initially, the correlation function of $S$ and AS modes becomes

$$
\begin{aligned}
\left\langle n_{S}(t) ; n_{A}(t)\right\rangle= & A(t)+\sum_{\mathbf{k q}} B_{\mathbf{k q}}(t)\left\langle a_{\mathbf{k} V}^{\dagger} a_{\mathbf{q} V}\right\rangle+\sum_{\mathbf{k} l \mathbf{p q}} C_{\mathbf{k} l \mathbf{p q}}(t) \\
& \times\left\langle a_{\mathbf{k} V}^{\dagger} a_{\mathbf{q} V} ; a_{l V}^{\dagger} a_{\mathbf{p} V}\right\rangle
\end{aligned}
$$

Here, parameters $A, B, C$ are functions of $u, \mathrm{v}, w$. Since the summations above can be converted into integrals involving 
phonon density of states, we see that if there are Van Hove singularities corresponding to the modes selected by Raman scattering, as in the case of recent experiments on the generation of nonclassical phonon states via Raman scatterings, ${ }^{7}$ then the correlation of $S$ and AS modes will be determined strongly by that mode. If this is not the case, then one can still expect domination of the modes obeying Raman selection rules. Then for that mode the random phase approximation permits us to write ${ }^{14}$

$$
\begin{gathered}
\left\langle n_{S}(t)\right\rangle=\left|\mathrm{v}_{S}(t)\right|^{2}+\left|w_{S}^{\prime}\right|^{2}\left(1+n_{V}\right), \\
\left\langle n_{A}(t)\right\rangle=\left|u_{A}(t)\right|^{2}+\left|w_{A}^{\prime}\right|^{2} n_{V}, \\
\left\langle n_{S}(t) ; n_{A}(t)\right\rangle=A^{\prime}(t)+B^{\prime}(t) n_{V}+C^{\prime}(t) V\left(n_{V}\right),
\end{gathered}
$$

in which the momentum label corresponding to the relevant mode is fixed and dropped for the notational simplicity and primed parameters evaluated at that mode. It is possible to argue by the results above that a measurement of the correlation between $S$ and AS can be utilized to determine the variance of vibration modes, which we usually consider as phonons here, provided one knows the mean number of such modes initially. The latter information can be determined by either one of the first two relations in Eq. (12) after measurement of radiation mode intensities. Also, measurement of radiation mode intensities and the knowledge of initial phonon number allow one to keep track of the evolution of mean phonon number through the Manley-Rowe relations given by Eq. (2). Interestingly, since the mean number of phonons with nonclassical distributions deviate significantly from that of Bose-Einstein distribution, it might be possible to find some traces of nonclassicality even here. However, in order to classify the distribution of phonons strictly it would still be necessary to find the next moment of the distribution, in other words the variance of phonons.

Now, an explicit way of determining $u, \mathrm{v}, w$ parameters will be demonstrated for the case of a single phonon mode. Because of the three-dimensional operator space in this situation, eigenvalues $E_{l}$ are found to be as the roots of the cubic equation,

$$
\begin{aligned}
E^{3}+ & 3 \omega_{V} E^{2}-\left[\omega_{R}^{2}-3 \omega_{V}^{2}+\left(\left|g^{A}\right|^{2}-\left|g^{S}\right|^{2}\right)\right] E \\
& +\left[\left|g^{S}\right|^{2}\left(\omega_{R}+\omega_{V}\right)+\left|g^{A}\right|^{2}\left(\omega_{R}-\omega_{V}\right)\right] \\
& +\omega_{V}\left(\omega_{V}^{2}-\omega_{R}^{2}\right)=0 .
\end{aligned}
$$

Introducing coefficients $P_{l}, Q_{l}$ as

$$
\begin{gathered}
P_{l}=-\frac{\left(E_{l}+\omega_{V}\right)\left(E_{l}+\omega_{R}+\omega_{V}\right)+\left|g^{S}\right|^{2}-\left|g^{A}\right|^{2}}{2 g^{S} \omega_{R}}, \\
Q_{l}=-\frac{g^{S} P_{l}+E_{l}+\omega_{V}}{g^{A *}},
\end{gathered}
$$

we write the field operators as

$$
\hat{a}_{S}^{\dagger}(t)=\sum_{l} P_{l} A_{l} e^{i E_{l} t}
$$

$$
\hat{a}_{A}(t)=\sum_{l} Q_{l} A_{l} e^{i E_{l} t}
$$

Common operator coefficients $A_{l}$ are determined in terms of the operators $a_{V}(0), a_{S}^{\dagger}(0), a_{A}(0)$ using the Cramer's rule $\hat{\mathcal{A}}_{l}=\operatorname{det}\left(D_{l}\right) / \operatorname{det}(D)$, where

$$
D=\left(\begin{array}{ccc}
1 & 1 & 1 \\
P_{1} & P_{2} & P_{3} \\
Q_{1} & Q_{2} & Q_{3}
\end{array}\right),
$$

and $D_{l}$ is the matrix obtained by replacing the elements in the $l$ th column of $D$ by the column vector $\left[\hat{a}_{V}(0), \hat{a}_{S}^{\dagger}(0), \hat{a}_{A}(0)\right]^{T}$. Thus, parameters $u, \mathrm{v}, w$ are determined in terms of interaction constants and the frequencies. More explicit expressions are too long and not very illuminating to reproduce here, but the above analysis is quite suitable for numerical computation when some experimental data is available. At that moment we shall content ourselves with more fundamental discussions only.

In order to give a brief discussion of the dependence of the correlation function in Eq. (9) on squeezing parameter and temperature, we consider an equilibrium distribution of vibration mode as of the squeezed thermal state with the following mean number and number variance: ${ }^{11}$

$$
\begin{gathered}
\left\langle n_{V}\right\rangle=\bar{n}_{V} \cosh 2 r+\sinh ^{2} r, \\
V_{0}\left(n_{V}\right)=\left(\bar{n}_{V}^{2}+\bar{n}_{V}\right) \cosh 4 r+\frac{1}{2} \sinh ^{2} 2 r,
\end{gathered}
$$

where $\bar{n}_{V}$ is the mean number of phonons according to BoseEinstein (BE) distribution and $r$ is the real squeezing parameter. When $r=0$, we recover the usual BE distribution. According to Eq. (10) the $S$ and AS correlations increases with variance of phonons. And since both the $n_{V}$ and the $V\left(n_{V}\right)$ increases with temperature due to Eq. (12), we see that temperature enforces stronger correlations of $S$ and AS modes. However, we need to put a word of caution here, since the fluctuations that are determined by the self-correlations of the modes also increase with the temperature. In order to represent this competition, one can consider the crosscorrelation function defined by ${ }^{24}$

$$
C_{S-A S}=\frac{\left\langle n_{S}, n_{A}\right\rangle}{\sqrt{V\left(n_{S}\right) V\left(n_{A}\right)}} .
$$

Since the denominator can be expressed in a similar structure as with the correlation function in Eq. (10), the crosscorrelation function will eventually saturate at high temperatures and at high-squeezing parameters. Therefore, at high temperatures thermal fluctuations becomes important but not more important than in any typical quantum measurement. An estimation for a typical ionic crystal, for example, shows that the level of quantum fluctuations of phonon number exceeds that of thermal fluctuations below $30-50 \mathrm{~K} .{ }^{8,9}$ We also see through Eq. (10) and Eq. (12) that $S$ and AS correlation increases with the squeezing parameter $r$.

Finally, we examine the time range of validity for the parametric approximation. For that aim, we consider the Hamiltonian given in Eq. (1) for the case of perfect coupling 
of single modes. Let us suppress the momentum within the mode labels $R, S, V, A$ and calculate $a_{R}(t)$ for times close to the beginning of interaction. ${ }^{25} \mathrm{Up}$ to the second order, we get

$$
\begin{aligned}
a_{R}(t)= & e^{-i \omega_{R} t}\left[a_{R}+i t\left(M^{S *} a_{S} a_{V}+M^{A *} a_{A} a_{V}^{\dagger}\right)\right. \\
& \left.-\frac{1}{2} t^{2}\left(\left|M^{S}\right|^{2} \nu+\left|M^{A}\right|^{2} \mu\right)\right],
\end{aligned}
$$

where $\nu=a_{R}\left(n_{S}+n_{V}+1\right), \mu=a_{R}\left(n_{A}-n_{V}\right)$. Here, operators at $t=0$ are those without time arguments. Then, we calculate that the mean number and the variance of pump photons for $S$ and AS modes are in vacuum states initially as

$$
\begin{aligned}
n_{R}(t) & =n_{R}-t^{2}\left[\left|M^{S}\right|^{2} n_{R}\left(1+n_{V}\right)+\left|M^{A}\right|{ }^{2} n_{R} n_{S}\right], \\
V\left[n_{R}(t)\right]= & V\left(n_{R}\right)+2 t^{2}\left(| M ^ { S } | ^ { 2 } \left\{V\left(n_{R}\right)\left(1+n_{V}\right)+n_{R}\left(1+n_{V}\right)\right.\right. \\
& \left.\left.+\left|M^{A}\right|^{2}\left[V\left(n_{R}\right) n_{V}-n_{R} n_{V}\right]\right\}\right) .
\end{aligned}
$$

In these equations the averaging symbol, \langle\rangle , is not shown. Using the relation $V\left(n_{R}\right)=n_{R}$ for a coherent field, we find the time ranges $t \ll \tau_{1}, \tau_{2}$, for which the field intensity and the variance remain close to their initial values, as

$$
\begin{gathered}
\tau_{1}=\frac{1}{\left|M^{S}\right|^{2}\left(1+n_{V}\right)+\left|M^{A}\right|^{2} n_{V}}, \\
\tau_{2}=\frac{1}{4\left|M^{S}\right|^{2}\left(1+n_{V}\right)} .
\end{gathered}
$$

Clearly, we see a rescaling of the time range of the usual time range of parametric approximation. At low temperatures $n_{V} \approx 0$, and thus $\tau_{2}=(1 / 4) \tau_{1}$ shows a reduction of time range to $1 / 4$ of the typical range of parametric approximation. As an estimation, we may take $g^{S} \approx 10^{7} \mathrm{~Hz},{ }^{25}$ giving time ranges as $\tau_{1}=10$ fs and $\tau_{2}=2.5$ fs. These ranges are readily available due to the remarkable recent developments in the field of femtosecond spectroscopy. ${ }^{26,27}$

\section{CONCLUSION}

Summing up our results, we should stress that the measurement of Stokes-anti-Stokes correlations looks like a reasonable method for detecting the number variance of a Raman-active vibration mode in solids. The most interesting and crucial fact is that the above method permits us to determine the number variance at thermal equilibrium, in other words, the variance just before the application of the pump beam. The phonon subsystem could be in a nonclassical state due to an interaction providing necessary correlations among phonons before the pump beam is applied. That interaction could be some anharmonic coupling with the heat bath, polaron, or polariton mechanisms. Since these mechanisms are usually weaker than the first-order Raman effect, after the application of the pump beam, dynamics of the phonon system is governed mainly by the Raman effect. Therefore, initial nonclassical state of phonons and nonclassical effects like squeezing, which require that phase coherence might be destroyed. That is why we have determined the general and fundamental formula given by Eq. (4) in terms of the initial state of phonons and showed that under certain conditions it provides direct information on the initial, thermal equilibrium variance of phonons. Analyzing those conditions of applicability, we propose that at liquid-N temperatures, using an intense coherent beam of ultrafast laser source such as Ti-sapphire as a pump for a Raman-active medium, one can measure the number correlation of the scattered Stokes and anti-Stokes modes and the mean photon numbers in these modes simultaneously by some photon counters, in order to determine the number variance of the vibration mode at equilibrium. The measurement can be realized through the use of a homodyne-type scheme ${ }^{13}$ in which the $S$ and AS photons are counted by two different detectors connected with a computer fixing the simultaneous arrival of the $S$ and AS photons. It is also shown that when the vibration mode is in a squeezed state then an increase in the correlation of the Stokes and anti-Stokes modes occurs.

The case of a multimode pump, important for ultrashort pulses, can be handled easily for materials that involve a strongly preferred phonon mode due to a Van Hove singularity in the frequency range of the pump, by an appropriate calculation of the effective coupling constants defined by Eq. (7), which in turn modify only the coefficients $A^{\prime}, B^{\prime}, C^{\prime}$ in Eq. (12). Thus our conclusions should also be valid in this case. For materials in which such phonon modes are many or do not exist at all, then application of a multimode pump and measurement of the Stokes-anti-Stokes correlation would still provide information on multimode phonon correlations according to the general formula Eq. (11). This is a valuable knowledge to classify a possible nonclassical multimode state of phonons like a multimode squeezed state.

So far, the best achievement in squeezing of phonons is reported to be $0.01 \%{ }^{7}$ provided by second-order Raman scattering. We would like to emphasize that this is not the squeezing parameter $r$ of Eq. (12) but related to $V\left(n_{V}\right)$. Hence, the change in the Stokes-anti-Stokes correlations we expect to be in the same order. There are other mechanisms that result in nonclassical excitations in solids with different expressions and larger values for $r$ and $V(n)$. In fact, squeezing parameter reflects the strength of interaction preparing the nonclassical state of these excitations, ${ }^{5}$ which is the initial phonon state in our scheme. The example of optical polariton that we have discussed in the Introduction, provides a two-mode squeezed state with squeezing parameter in the range $r \sim 0.1-0.01 \mathrm{in} \mathrm{CuCl}^{4}{ }^{4}$ Therefore, such a measurement with the ultrafast Raman correlation spectroscopy should not be too challenging and looks promising in our opinion.

Let us finally note that the case of molecular Raman spectroscopy can also be treated with a similar formalism to get information on the quantum statistics of populations of molecular energy levels.

\section{ACKNOWLEDGMENTS}

We acknowledge useful discussions with Professor A. Bandilla and Professor V. Rupasov. 
${ }^{1}$ D. Stoler, Phys. Rev. D 1, 3217 (1970); 4, 1925 (1971).

${ }^{2}$ N. N. Bogolubov, J. Phys. (Moscow) 11, 23 (1947).

${ }^{3}$ D. Robinson, Commun. Math. Phys. 1, 159 (1965).

${ }^{4}$ M. Artoni and J. L. Birman, Phys. Rev. B 44, 3736 (1991); Opt. Commun. 104, 319 (1994).

${ }^{5}$ X. Hu and F. Nori, Phys. Rev. B 53, 2419 (1996); Phys. Rev. Lett. 76, 2294 (1996).

${ }^{6}$ Ö. Müstecaplioğlu and A. S. Shumovsky, Appl. Phys. Lett. 70, 3489 (1997).

${ }^{7}$ G. A. Garrett, A. G. Rojo, A. K. Sood, J. F. Whitaker, and R. Merlin, Science 175, 1638 (1997).

${ }^{8}$ A. S. Shumovsky, in Quantum Optics and the Spectroscopy of Solids, edited by T. Hakioğlu and A. S. Shumovsky (Kluwer, Dordrecht, 1997).

${ }^{9}$ A. V. Chizhov, R. G. Nazmitdinov, and A. S. Schumovsky, Quantum Opt. 3, 1 (1991); Mod. Phys. Lett. B 7, 1233 (1993).

${ }^{10}$ T. Altanhan and B. S. Kandemir, J. Phys.: Condens. Matter 5, 6729 (1993).

${ }^{11}$ M. S. Kim, F. A. M. de Oliveira, and P. L. Knight, Phys. Rev. A 40, 2494 (1989).

${ }^{12}$ O. Madelung, Introduction to Solid-State Theory (Springer, New York, 1978).

${ }^{13}$ L. Mandel and E. Wolf, Optical Coherence and Quantum Optics (Cambridge University Press, Cambridge, 1994).

${ }^{14}$ H. Haug and S. W. Koch, Quantum Theory of the Optical and
Electronic Properties of Semiconductors (World Scientific, Hong Kong, 1992).

${ }^{15}$ A. Shumovsky and B. Tanatar, Phys. Rev. A 48, 4735 (1993); Phys. Lett. A 182, 411 (1993).

${ }^{16}$ L. Allen and J. H. Eberly, Optical Resonance and Two-Level Atoms (Dover, New York, 1987).

${ }^{17}$ Y. R. Shen, The Principles of Non-Linear Optics (Wiley, New York, 1984).

${ }^{18}$ D. F. Walls, J. Phys. A 6, 496 (1973).

${ }^{19}$ J. Peřina, Opt. Acta 28, 325 (1981); 28, 1529 (1981).

${ }^{20}$ S. Carusotto, Phys. Rev. A 40, 1848 (1989).

${ }^{21}$ D. F. Walls, Z. Phys. 237, 224 (1970).

${ }^{22} \mathrm{R}$. Loudon, The Quantum Theory of Light (Oxford University Press, Oxford, 1983).

${ }^{23}$ D. Chowdhury, Spin Glasses and Other Frustrated Systems (Princeton University Press, Princeton, NJ, 1986).

${ }^{24}$ C. K. Law, L. Wang, and J. H. Eberly, Phys. Rev. A 45, 5089 (1992).

${ }^{25}$ J. Peřina, Quantum Statistics of Linear and Nonlinear Optical Phenomena (Reidel, Dordrecht, 1984).

${ }^{26}$ J.-C. Dies and W. Rudolph, Ultrafast Laser Pulse Phenomena (Academic Press, San Diego, 1996).

${ }^{27}$ J. Shah, Ultrafast Spectroscopy of Semiconductors and Semiconductor Nanostructures (Springer, Berlin, 1996). 